International Journal of Pure and Applied Mathematics

Volume 94 No. $4 \quad 2014,541-549$

ISSN: 1311-8080 (printed version); ISSN: 1314-3395 (on-line version)

url: http://www.ijpam.eu

doi: http://dx.doi.org/10.12732/ijpam.v94i4.10

\title{
OPTIMAL CONVEX COMBINATION BOUNDS OF THE CONTRAHARMONIC AND HARMONIC MEANS FOR THE SEIFFERT MEAN
}

\author{
Gao Shaoqin $^{1 \S}$, Song Lingling ${ }^{2}$, You Mengna ${ }^{3}$ \\ ${ }^{1,2,3}$ College of Mathematics and Computer Sciences \\ Hebei University \\ Baoding, 071002, P.R. CHINA
}

Abstract: We find the greatest value $\alpha$ and the least value $\beta$ such that the double inequality

$$
\alpha C(a, b)+(1-\alpha) H(a, b)<P(a, b)<\beta C(a, b)+(1-\beta) H(a, b)
$$

holds for all $a, b>0$ with $a \neq b$. Here $\mathrm{C}(a, b), \mathrm{H}(a, b)$ and $\mathrm{P}(a, b)$ denote the contraharmonic, harmonic, and the Seiffert means of two positive numbers $a$ and $b$ respectively.

AMS Subject Classification: 26D15

Key Words: optimal convex combination bound, contraharmonic mean, harmonic mean, the Seiffert means

\section{Introduction}

For $a, b>0$ with $a \neq b$ the Seiffert means $\mathrm{P}(a, b)$ was introduced by Seiffert $[1,2]$ as follows:

$$
P(a, b)=\frac{a-b}{4 \arctan (\sqrt{a / b})-\pi} .
$$

Recently, the inequalities for means have been the subject of intensive re-

Received: April 7, 2014

(C) 2014 Academic Publications, Ltd.

${ }^{\S}$ Correspondence author 
search $[3,8]$. In particularly, many remarkable inequalities for the Seiffert mean can be found in the literature $[4,6,8]$.

Let $H(a, b)=\frac{2 a b}{a+b}, G(a, b)=\sqrt{a b}, \mathrm{~L}(a, b)=\frac{b-a}{\ln b-\ln a}, P(a, b)=\frac{a-b}{4 \arctan \sqrt{\frac{a}{b}}-\pi}$, $I(a, b)=\frac{1}{e}\left(\frac{b^{\mathrm{b}}}{a^{\mathrm{a}}}\right)^{\frac{1}{\mathrm{~b}-\mathrm{a}}}, A(a, b)=\frac{a+b}{2}, \mathrm{~T}(a, b)=\frac{2\left(a^{2}+a b+b^{2}\right)}{3(a+b)}$ and $C(a, b)=\frac{a^{2}+b^{2}}{a+b}$ be the harmonic, geometric, logarithmic, Seiffert, identric, arithmetic, centroidal and contraharmonic means of two positive real numbers $a$ and $b$ with $a \neq b$. Then

$$
\begin{aligned}
& \min \{a, b\}<H(a, b)<G(a, b)<L(a, b)<\mathrm{P}(a, b)<I(a, b)<A(a, b) \\
& <T(a, b)<S(a, b)<C(a, b)<\max \{a, b\} .
\end{aligned}
$$

In [1], Seiffert proved

$$
L(a, b)<P(a, b)<I(a, b)
$$

for all $a, b>0$ with $a \neq b$.

The following bounds for the Seiffert mean $P(a, b)$ in terms of the power mean $M_{r}(a, b)=\left(\left(a^{r}+b^{r}\right) / 2\right)^{1 / r}(r \neq 0)$ were presented by Jagers in [7]:

$$
M_{1 / 2}(a, b)<P(a, b)<M_{2 / 3}(a, b)
$$

for all $a, b>0$ with $a \neq b$.

$H \ddot{a} s t \ddot{o}[8]$ found the sharp lower bound for the Seiffert mean as follow:

$$
M_{\log 2 / \log \pi}(a, b)<P(a, b)
$$

for all $a, b>0$ with $a \neq b$.

In [3], Seiffert proved

$$
P(a, b)>\frac{3 A(a, b) G(a, b)}{A(a, b)+2 G(a, b)}
$$

and

$$
P(a, b)>\frac{2}{\pi} A(a, b)
$$

for all $a, b>0$ with $a \neq b$.

In [4], the authors found the greatest value $\alpha$ and the least value $\beta$ such that the double inequality

$$
\alpha A(a, b)+(1-\alpha) H(a, b)<P(a, b)<\beta A(a, b)+(1-\beta) H(a, b)
$$

for all $a, b>0$ with $a \neq b$. 
The purpose of the present paper is to find the greatest value $\alpha$ and the least value $\beta$ such that the double inequality

$$
\alpha C(a, b)+(1-\alpha) H(a, b)<P(a, b)<\beta C(a, b)+(1-\beta) H(a, b)
$$

holds for all $a, b>0$ with $a \neq b$.

\section{Main Result}

\subsection{Theorem}

The double inequality

$$
\alpha C(a, b)+(1-\alpha) H(a, b) \leq P(a, b) \leq \beta C(a, b)+(1-\beta) H(a, b)
$$

holds for all $a, b>0$ with $a \neq b$ if and only if $\alpha \leq \frac{1}{\pi}$ and $\beta \geq \frac{5}{12}$.

Proof. Firstly, we prove that

$$
\begin{gathered}
P(a, b)<\frac{5}{12} C(a, b)+\frac{7}{12} H(a, b), \\
P(a, b)>\frac{1}{\pi} C(a, b)+\left(1-\frac{1}{\pi}\right) H(a, b),
\end{gathered}
$$

for all $a, b>0$ with $a \neq b$.

Without loss of generality, we assume $a>b>0$. Let $t=\sqrt{\frac{a}{b}}>1$ and $q \in\left\{\frac{1}{\pi}, \frac{5}{12}\right\}$. Then (1) leads to

$$
\begin{aligned}
\frac{1}{b} & {[q C(a, b)+(1-q) H(a, b)-P(a, b)] } \\
& =\frac{q t^{4}+2(1-q) t^{2}+q}{\left(t^{2}+1\right)(4 \operatorname{arctant}-\pi)} f(t)
\end{aligned}
$$

where

$$
f(t)=4 \arctan t-\pi-\frac{t^{4}-1}{q t^{4}+2(1-q) t^{2}+q} .
$$

Simple computations lead to

$$
\lim _{t \rightarrow 1+} f(t)=0
$$




$$
\begin{gathered}
\lim _{t \rightarrow+\infty} f(t)=\pi-\frac{1}{q} . \\
f^{\prime}(t)=\frac{4}{\left(t^{2}+1\right)\left[q t^{4}+2(1-q) t^{2}+q\right]^{2}} g_{1}(t),
\end{gathered}
$$

where

$$
\begin{aligned}
g_{1}(t)= & q^{2} t^{8}-(1-q) t^{7}+4 q(1-q) t^{6}-(1+q) t^{5} \\
& +2\left(3 q^{2}-4 q+2\right) t^{4}-(1+q) t^{3} \\
& +4 q(1-q) t^{2}-(1-q) t+q^{2} .
\end{aligned}
$$

Now we divide the proof into two cases:

Case 1. If $q=\frac{5}{12}$. (17) leads to

$$
g_{1}(t)=\frac{(t-1)^{4}}{144}\left(25 t^{4}+16 t^{3}+54 t^{2}+16 t+25\right)>0
$$

for $t>1$. (18) and (16) imply $f^{\prime}(t)>0$, thus $f(t)$ is strictly increasing for $t>1$. Then inequality (10) follows from (12)-(14).

Case 2. If $q=\frac{1}{\pi}$, then from (17) we get

$$
\begin{gathered}
\lim _{t \rightarrow 1+} g_{1}(t)=0, \\
\lim _{t \rightarrow+\infty} g_{1}(t)=+\infty . \\
g_{1}^{\prime}(t)=8 q^{2} t^{7}-7(1-q) t^{6}+24 q(1-q) t^{5}-5(1+q) t^{4} \\
+8\left(3 q^{2}-4 q+2\right) t^{3}-3(1+q) t^{2}+8 q(1-q) t-(1-q), \\
\lim _{t \rightarrow 1+} g_{1}{ }^{\prime}(t)=0, \\
\lim _{t \rightarrow+\infty} g_{1}{ }^{\prime}(t)=+\infty .
\end{gathered}
$$




$$
\begin{gathered}
g_{1}^{\prime \prime}(t)=56 q^{2} t^{6}-42(1-q) t^{5}+120 q(1-q) t^{4}-20(1+q) t^{3} \\
+24\left(3 q^{2}-4 q+2\right) t^{2}-6(1+q) t+8 q(1-q), \\
\lim _{t \rightarrow 1+} g_{1}^{\prime \prime}(t)=48 q-20=\frac{48}{\pi}-20<0, \\
\lim _{t \rightarrow+\infty} g_{1}^{\prime \prime}(t)=+\infty . \\
g_{1}^{\prime \prime \prime}(t)=6\left[\frac{56}{\pi^{2}} t^{5}-35\left(1-\frac{1}{\pi}\right) t^{4}+\frac{80}{\pi}\left(1-\frac{1}{\pi}\right) t^{3}-10\left(1+\frac{1}{\pi}\right) t^{2}\right. \\
\left.+\left(\frac{3}{2 \pi^{2}}-\frac{2}{\pi}+1\right) t-\left(1+\frac{1}{\pi}\right)\right]
\end{gathered}
$$

Let

$$
\begin{aligned}
g_{2}(t)=\frac{56}{\pi^{2}} t^{5}-35\left(1-\frac{1}{\pi}\right) t^{4}+\frac{80}{\pi}\left(1-\frac{1}{\pi}\right) t^{3} & -10\left(1+\frac{1}{\pi}\right) t^{2} \\
& +\left(\frac{3}{2 \pi^{2}}-\frac{2}{\pi}+1\right) t-\left(1+\frac{1}{\pi}\right) .
\end{aligned}
$$

Then

$$
\begin{gathered}
\lim _{t \rightarrow 1+} g_{2}(t)=\frac{72}{\pi}-30<0, \\
\lim _{t \rightarrow+\infty} g_{2}(t)=+\infty . \\
g_{2}{ }^{\prime}(t)=\frac{280}{\pi^{2}} t^{4}-140\left(1-\frac{1}{\pi}\right) t^{3}+\frac{240}{\pi}\left(1-\frac{1}{\pi}\right) t^{2} \\
\left.\lim _{t \rightarrow 1+} g_{2}{ }^{\prime}(t)=\frac{64}{\pi^{2}}+\frac{328}{\pi}-144<0, \frac{1}{\pi}\right) t+16\left(1+\frac{3}{2 \pi^{2}}-\frac{2}{\pi}\right), \\
\lim _{t \rightarrow+\infty} g_{2}{ }^{\prime}(t)=+\infty .
\end{gathered}
$$




$$
g_{2}^{\prime \prime}(t)=20\left[\frac{56}{\pi^{2}} t^{3}-21\left(1-\frac{1}{\pi}\right) t^{2}+\frac{24}{\pi}\left(1-\frac{1}{\pi}\right) t-\left(1+\frac{1}{\pi}\right)\right]=20 g_{3}(t),
$$

where

$$
g_{3}(t)=\frac{56}{\pi^{2}} t^{3}-21\left(1-\frac{1}{\pi}\right) t^{2}+\frac{24}{\pi}\left(1-\frac{1}{\pi}\right) t-\left(1+\frac{1}{\pi}\right) .
$$

Therefore,

$$
\begin{gathered}
\lim _{t \rightarrow 1+} g_{3}(t)=\frac{32}{\pi^{2}}+\frac{44}{\pi}-22<0, \\
\lim _{t \rightarrow+\infty} g_{3}(t)=+\infty . \\
g_{3}{ }^{\prime}(t)=6\left[\frac{28}{\pi^{2}} t^{2}-7\left(1-\frac{1}{\pi}\right) t+\frac{4}{\pi}\left(1-\frac{1}{\pi}\right)\right]=6 g_{4}(t),
\end{gathered}
$$

where

$$
g_{4}(t)=\frac{28}{\pi^{2}} t^{2}-7\left(1-\frac{1}{\pi}\right) t+\frac{4}{\pi}\left(1-\frac{1}{\pi}\right) .
$$

By simple computations, we have

$$
\begin{gathered}
\lim _{t \rightarrow 1+} g_{4}(t)=\frac{24}{\pi^{2}}+\frac{11}{\pi}-7<0, \\
\lim _{t \rightarrow+\infty} g_{4}(t)=+\infty . \\
g_{4}{ }^{\prime}(t)=\frac{56}{\pi^{2}} t-7\left(1-\frac{1}{\pi}\right), \\
\lim _{t \rightarrow 1+} g_{4}{ }^{\prime}(t)=\frac{56}{\pi^{2}}-7\left(1-\frac{1}{\pi}\right)>0, \\
g_{4}{ }^{\prime \prime}(t)=\frac{56}{\pi^{2}}>0 .
\end{gathered}
$$

From (44) and (43) we clearly see that $g_{4}{ }^{\prime}(t)>0$ for $t>1$, hence $g_{4}(t)$ is strictly increasing in $[1,+\infty)$. It follows from (40)and (41) together with the monotonicity of $g_{4}(t)$ that there exists $\lambda_{1}>1$ such that $g_{4}(t)<0$ for $t \in\left[1, \lambda_{1}\right)$ and $g_{4}(t)>0$ for $t \in\left(\lambda_{1},+\infty\right)$, hence from $(38) g_{3}(t)$ is strictly decreasing in $\left[1, \lambda_{1}\right]$ and strictly increasing in $\left[\lambda_{1},+\infty\right)$. 
From (36) and (37) together with the monotonicity of $g_{3}(t)$ we know that there exists $\lambda_{2}>1$ such that $g_{3}(t)<0$ for $t \in\left[1, \lambda_{2}\right)$ and $g_{3}(t)>0$ for $t \in\left(\lambda_{2},+\infty\right)$, hence from (34) $g_{2}{ }^{\prime}(t)$ is strictly decreasing in $\left[1, \lambda_{2}\right]$ and strictly increasing in $\left[\lambda_{2},+\infty\right)$.

From (32) and (33) together with the monotonicity of $g_{2}{ }^{\prime}(t)$ we can see that there exists $\lambda_{3}>1$ such that $g_{2}(t)$ is strictly decreasing in $\left[1, \lambda_{3}\right]$ and strictly increasing in $\left[\lambda_{3},+\infty\right)$. It follows from $(27)(29)$ and (30) together with the monotonicity of $g_{2}(t)$ we clearly see there exists $\lambda_{4}>1$ such that $g_{1}{ }^{\prime \prime}(t)$ is strictly decreasing in $\left[1, \lambda_{4}\right]$ and strictly increasing in $\left[\lambda_{4},+\infty\right)$.

From (25) and (26) together with the monotonicity of $g_{1}{ }^{\prime \prime}(t)$ we can see that there exists $\lambda_{5}>1$ such that $g_{1}{ }^{\prime}(t)$ is strictly decreasing in $\left[1, \lambda_{5}\right]$ and strictly increasing in $\left[\lambda_{5},+\infty\right)$. From (22) and (23) together with the monotonicity of $g_{1}{ }^{\prime}(t)$ we clearly see there exists $\lambda_{6}>1$ such that $g_{1}(t)$ is strictly decreasing in $\left[1, \lambda_{6}\right]$ and strictly increasing in $\left[\lambda_{6},+\infty\right)$. Then (16) (19) and (20) imply that there exists $\lambda_{7}>1$ such that $f(t)$ is strictly decreasing in $\left[1, \lambda_{7}\right]$ and strictly increasing in $\left[\lambda_{7},+\infty\right)$. Note that (15) becomes

$$
\lim _{t \rightarrow+\infty} f(t)=0
$$

for $p=\frac{1}{\pi}$.

It follows from (14) and (45) together with the monotonicity of $f(t)$ that

$$
f(t)<0
$$

for $t>1$.

Therefore, inequality (11) follows from (12) and (13) together with (46).

Secondly, we prove that $\frac{5}{12} C(a, b)+\frac{7}{12} H(a, b)$ is the best possible upper convex combination bound of contraharmonic and harmonic means for the Seiffert mean $P(a, b)$.

For any $t>1$ and $\alpha \in R$, we have

$$
\begin{aligned}
& \alpha C\left(t^{2}, 1\right)+(1-\alpha) H\left(t^{2}, 1\right)-P\left(t^{2}, 1\right) \\
= & \frac{q\left(t^{4}+1\right)}{t^{2}+1}+(1-q) \frac{2 t^{2}}{t^{2}+1}-\frac{\left(t^{2}-1\right)}{4 \operatorname{arctant}-\pi} \\
= & \frac{h(t)}{\left(t^{2}+1\right)(4 \operatorname{arctant}-\pi)},
\end{aligned}
$$

where

$$
h(t)=\left[q t^{4}+q+2(1-q) t^{2}\right](4 \arctan t-\pi)-\left(t^{4}-1\right) .
$$


It follows from (2.39) that

$$
\begin{gathered}
h(1)=h^{\prime}(1)=h^{\prime \prime}(1)=0, \\
h^{\prime \prime \prime}(1)=4(12 \alpha-5) .
\end{gathered}
$$

If $\alpha<\frac{5}{12}$, then (2.41) leads to

$$
h^{\prime \prime \prime}(1)<0 \text {. }
$$

From (2.42) and the continuity of $h^{\prime \prime \prime}(t)$ we see that there exists $\delta=\delta(\alpha)>0$ such that

$$
h^{\prime \prime \prime}(t)<0
$$

for $t \in[1,1+\delta)$. Then (2.40) and (2.43) imply that

$$
h(t)<0
$$

for $t \in[1,1+\delta)$.

Therefore, $\alpha C\left(t^{2}, 1\right)+(1-\alpha) H\left(t^{2}, 1\right)<P\left(t^{2}, 1\right)$ for $t \in(1,1+\delta)$ follows from (2.38) and (2.44).

Finally, we prove that $\frac{1}{\pi} C(a, b)+\left(1-\frac{1}{\pi}\right) H(a, b)$ is the best possible lower convex combination bound of contraharmonic and harmonic means for the Seiffert mean $P(a, b)$. In fact, for $\beta>\frac{1}{\pi}$, we have

$$
\lim _{x \rightarrow+\infty} \frac{\beta C(1, x)+(1-\beta) H(1, x)}{P(1, x)}=\pi \beta>1
$$

Inequality (2.45) implies that for any $\beta>\frac{1}{\pi}$ there exists $X=X(\beta)>1$ such that $\beta C(1, x)+(1-\beta) H(1, x)>P(1, x)$ for $x \in(X,+\infty)$.

\section{Acknowledgments}

This research is partly supported by the National Natural Science Foundation of China (11271106). 


\section{References}

[1] H. Seiffert,Problem 887,Nieuw Archief voor Wiskunde, vol.11, No.2 (1993),176-176.

[2] H. Seiffert, Aufgabe 16, Die Wurzel, no.29 (1995), 221-222.

[3] H. Seiffert, Ungleichungen für einen bestimmten mittelwert, Nieuw Archief voor Wiskunde, vol.13, No.2 (1995), 195-198.

[4] Y. Chu, Y. Qiu, M. Wang, G. Wang, The optimal convex combination bounds of arithmetic and harmonic means for the Seifferts mean, Journal of Inequalities and Applications, Article ID 436457, dio: 10.1155/436457,2010,7 pages .

[5] T. Hara, M. Uchiyama, S. Takahasi, A refinement of various mean inequalities, Journal of Inequalities and Applications, vol.2, No.4 (1998), 387-395.

[6] E. Neuman, J. Sändor, On the Schwab-Borchardt mean, Mathematica Pannonica, vol.17, No.1 (2006), 49-59.

[7] A. Jagers, Solution of problem 887, Nieuw Archief voor Wiskunde, 12(1994), 230-231.

[8] P. P. Hästö, Optimal inequalities between Seiffert's mean and power mean, Mathematical Inequalities and Applications, vol.7, No.1 (2004), 4753. 
